\title{
Nose-to-brain delivery of antiglioblastoma drugs embedded into lipid nanocarrier systems: status quo and outlook
}

\section{Fakhara Sabir, Ruba Ismail and Ildiko Csoka}

Q11

University of Szeged, Faculty of Pharmacy, Institute of Pharmaceutical Technology and Regulatory Affairs, H-6720 Szeged, Eötvös u. 6, Hungary

Q13 Glioblastoma (GBM) is one of the most devastating and deadly types of tumor. Among all the present treatment strategies, the utmost prerequisite is prolonged intervention at the malignant site. The ${ }_{014}$ blood-brain barrier (BBB) is the bottleneck in the delivery of anti-GBM drugs and invasive treatment comes with many pitfalls. This review will discuss the potential of embedding antitumor drugs into nanocarriers for intranasal delivery. Additionally, it emphasizes the significance of applying quality by design (QbD) methodology from the early development stages to ensure the high quality, safety and efficacy of the developed carrier system.

\section{Introduction}

Malignant gliomas (MGs) are the most lethal forms of primary central nervous system (CNS) malignancy, classified based on an augmenting level of undifferentiation, anaplasia and proliferation. WHO classified gliomas into four clinical grades: grade I (astrocytoma); grade II (diffuse astrocytoma, the most distinguished form); grade III (anaplastic variants of astrocytoma); Q15 and grade IV (glioblastoma) [1]. Pleomorphic glioblastoma is called glioblastoma multiforme (GBM) because these malignant cells show a discrepancy in structure and morphology [2]. The current treatment strategies for GBM include surgery, radiotherapy and chemotherapy. The focus of researchers to treat GBM is challenging because surgery and radiotherapy are not good options because of its topographically diffuse nature [3]. Eventually, understanding the pattern of spread of individual malignant cells over long distances and into parts of the brain is essential for patient survival. A present literature survey reveals that there are just a few available therapies that could significantly improve survival chances [4]. The circumvention of the blood-brain barrier (BBB) through straight intervention into insubstantial brain tissues can result in severe neurotoxicity and loss of brain key functionality. Consequently, there is a need to design a more specific and rational (noninvasive) approach to target GBM. It is also necessary to explore the potential differences in permeability

Corresponding author: Csoka, I. (csoka@pharm.u-szeged.hu) between the intact and malignant brain to overcome the challenges in brain targeting [5]. Figure 1 demonstrates the differences between barriers in the intact brain and in glioblastoma.

The intranasal route is a direct and simple approach, including many advantages of higher bioavailability, shorter onset of action, circumvention of systemic toxicity, noninvasiveness and clearance. Additionally, avoiding the BBB could significantly increase the concentration of the active pharmaceutical agent in the central nervous system (CNS). According to data present in the literature, pharmacological active agents can be delivered through the nasal cavity via the trigeminal and olfactory nerves. Drug permeation is basically dependent upon the key characteristics of an active agent or carrier, like its metabolic stability, solubility, residence time in the mucous layer and rate of mucociliary clearance [6].

The safety and toxicological evaluation of products delivered intranasally is of great importance. The prolonged contact of formulations containing cytotoxic materials can cause ciliotoxicity, tissue damage and irritation [7]. Therefore, regardless of the presence of carrier-free approaches for intranasal delivery, a carrier system could be useful to deliver chemotherapeutics via the intranasal route; among all delivery systems, nanoparticlebased carriers have been intensely studied for research imaging, treatment and diagnosis of brain tumors. Lipid-based colloidal systems, for example liposomes and solid lipid nanoparticles, increased drug transfer to the brain through the intranasal 


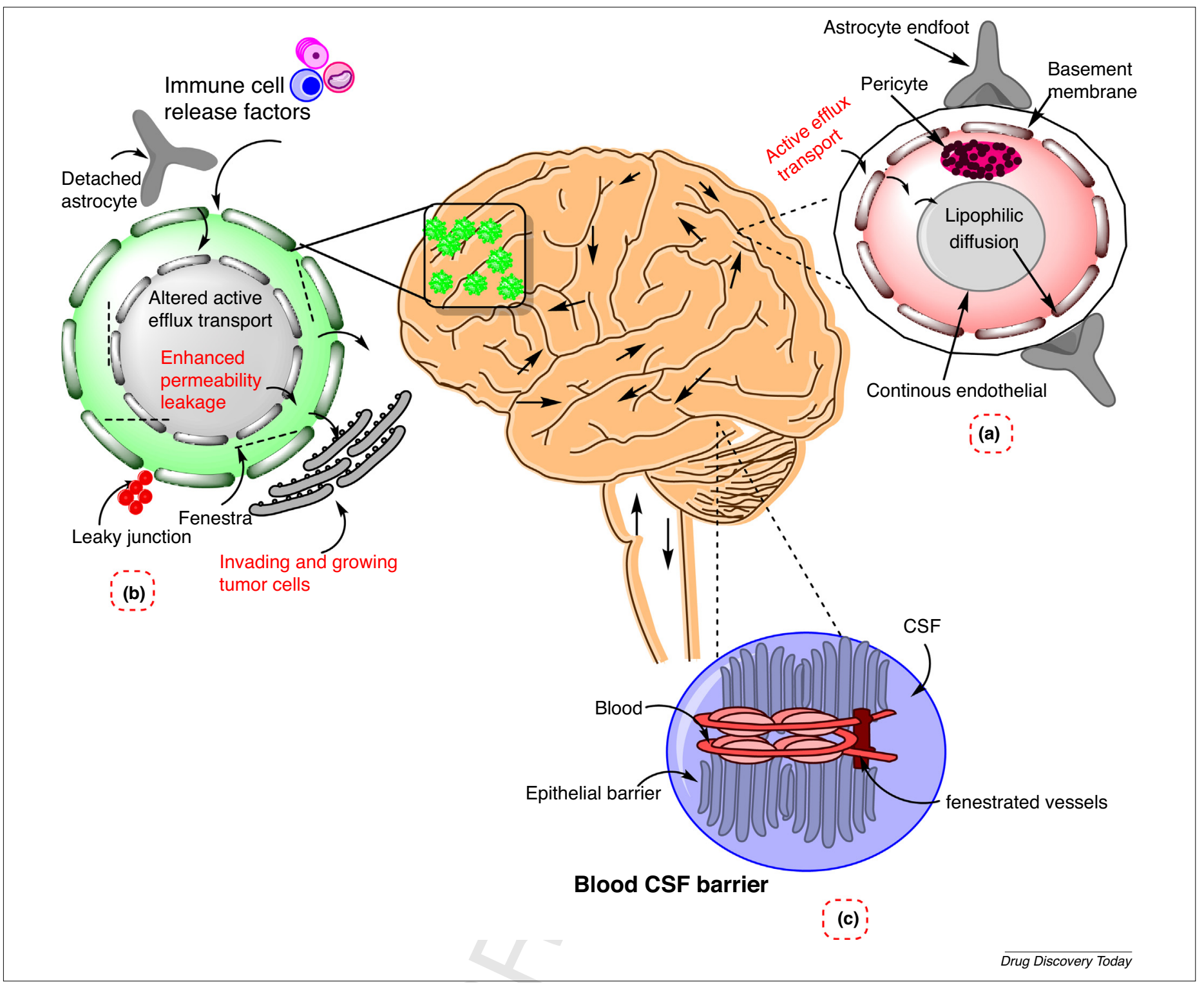

FIGURE 1

Q1 Challenges in blood to brain delivery in a brain tumor. The figure illustrates the comparison between barriers in normal brain and in glioblastoma multiforme (GBM). (a) Normal blood-brain barrier (BBB) composed of: astrocyte (role in morphology), pericyte, endothelial cells (role in tight junction structure and vasoregulation). (b) Blood-tumor barrier (deatched astrocyte, fenestra, leaky juctions - blood vessels that supply the tumor are leaky and incompletely formed but the healthy brain components are still present in the main region of GBM). (c) Blood-cerebrospinal-fluid (CSF) barrier (composed of a choroid plexus having epithelial cells and tight junctions, increased level of albumin in the CSF in GBM which might cause disturbance of the BBB or release from tumor).

route [8]. For instance, in vitro hemolysis and cytotoxicity studies of doxorubicin (DOX)-loaded liposomal nanoparticles were performed, which resulted in specificity and enhanced levels of drug accumulation in gliomas $[9,10]$. Colloidal nanocarrier systems [liposomes, solid lipid nanoparticles (SLNs), lipoproteins, lipoplexes, etc.] have shown clear amassing in gliomas but the shortening of noninvasive accumulation and retention evaluation tools could hinder monitoring the exact duration and location of nanoparticles within the brain [11]. This review will focus on the potential of lipid nanocarrier systems to deliver anticancer drugs via the intranasal route, as well as the significance of applying quality by design (QbD) software for the targeted delivery of cytotoxic materials via the intranasal route to maintain the Q16 safety and product profile.

\section{Strategies to circumvent the BBB: bottleneck in targeting glioblastoma}

Scientists have been working to develop versatile methods to circumvent the $\mathrm{BBB}$, which include the opening of the $\mathrm{BBB}$, intranasal delivery and penetration via the BBB by cellular internalization. The overexpression of receptors (like low-density lipoprotein, nicotinic acetylene choline, insulin-like growth factor (IGF), transferrin receptors, diphtheria toxin, leptin and scavenger receptor type B) has been reported on the BBB. The specific ligand functionalization and attachment can intervene in drug transport via the BBB. This precise and sensitive type of interaction between ligands and receptors governs receptor-mediated transport [12-14]. However, there are limitations in implementation of a functionalized or specific ligand attached moiety. First, it can lose 
its therapeutic activity; second, all present strategies are invasive and accumulation of drug cargos in the liver and other off-target Q17 sites governs its therapeutic efficacy [7]. Therefore, there is a need for noninvasive delivery approaches to achieve the best therapeutic goals.

\section{An alternative route of administration}

An alternative route of administration to CNS drug delivery is intranasal administration. The intranasal delivery (IND) route is a noninvasive, direct and more effective route of administration than intravenous (i.v.) delivery and can avoid the BBB together with systemic side effects. The IND pathways (trigeminal and olfactory pathways) in the nasal cavity are reasons for direct delivery to the brain and result in good pharmacokinetic/pharmacodynamic (PK/PD) profiles for CNS drugs. Drug delivery from the nose via the trigeminal pathway follows either axonal or endocytotic transport, whereas the olfactory pathway is further divided into intraneuronal and extraneuronal pathways. The intraneuronal pathway follows axonal transport and it takes hours or days Q18 for the API to reach the target site, whereas the extraneuronal path follows the perineural route and it just takes a few minutes to reach the target site $[15,16]$. Furthermore, this delivery route is a new approach for the delivery of potent active agents and for antineoplastic agents that can be loaded into nanocarriers to ensure a better safety profile. By using this route, nanoparticles can carry drugs easily to the target site, and can bypass the main barriers: the $\mathrm{BBB}$ and the blood-cerebrospinal-fluid barrier (BCSFB). There are many studies revealing better target delivery of CNS drugs via the Q19 intranasal route in contrast to i.v. administration. Schioth et al. reported that IGF-1, when given intranasally, had greater CNS efficacy when compared with i.v. intervention. Many other studies also showed that the intranasal delivery of the API led to better cure rates of CNS diseases, such as depression, autism, eating disorders, Parkinson's disease (PD) and Huntington's disease (HD), as well as various other diseases yet to be treated. Besides these advantages, there is a long list of factors that can limit the permeability of drug carriers via the intranasal route. Therefore, while designing intranasal formulations, the factors regarding the anatomy and the physiology of the nasal cavity should be considered. The vibrissae of the nasal vestibule and the transepithelial region of the atrium (narrowest region) are the parts that are the least permeable. By contrast, other parts like the superior, middle and inferior turbinate of the respiratory region are more permeable, whereas the specialized ciliated olfactory nerve cells of the olfactory region have direct access to the CSF. Table 1 explains how the structures of the nasal cavity affect permeability via the intranasal route $[16,17]$. By considering the crucial factors affecting drug delivery via the nasal cavity, a formulation can be designed for nose-to-brain delivery with increased permeation, low clearance and high mucoadhesion by the application of the QbD [18].

\section{Nanocarrier systems for nose-to-brain delivery}

The safety data for intranasal formulations are of great importance. Nanoparticles have the potential to improve nose-to-brain delivery because they have can avoid enzymatic degradation and transport from P-glycoprotein (P-gp) efflux proteins. These carrier systems can enhance therapeutic brain delivery by using bioadhesive materials and also by opening the closed junctions of the nasal epithelial membrane. The transport of nanoparticles through the intranasal route takes place via olfactory neurons by endocytotic or neuronal pathways. The confocal microscopy study of polystyrene nanoparticles reported that nanocarriers within the range $20-200 \mathrm{~nm}$ can follow clathrin-coated pits; however, nanoparticles in the size range $200-1000 \mathrm{~nm}$ can be transported via caveolae-mediated endocytosis $[7,15]$.

Nanoparticles can also follow the transport from endothelial cells to olfactory neurons via endocytosis or pinocytosis and move along the axon. For this transport pathway, the size of nanoparticles should be within the diameter of the axon, which is up to $100-700 \mathrm{~nm}$. Therefore, the intranasal delivery of nanoparticles could be a promising choice for targeting life-threatening diseases like glioblastoma. Regarding the carrier systems, the $\mathbf{Q 2 0}$ essential classes of nanoparticles that are at the center of focus for brain targeting include polymeric nanoparticles such as micelles, iron oxide nanoparticles, gold nanoparticles, quantum dots and lipid-based nanoparticles like nano lipid carriers (NLCs), SLNs, liposomes, lipoplexes and lipoproteins [19]. In general, nanopar- 021 ticles can induce toxicity depending upon their internalization site and composition. It is also reported that nanoparticles can induce inflammation, DNA damage and oxidative stress. The IND of metal nanoparticles into brain is relatively well known for harmful neurological effects. The extensive exposure of metal nanoparticles can cause serious damage and even can lead to diseases such as Alzheimer's disease (AD) and parkinsonism. The present study is relevant to lipid nanoparticles that are most biocompatible and least toxic in nature. Clearance of nanoparticles from the brain occurs via the mononuclear phagocytes system (MPS). The aspects of toxicity and clearance via macrophages are not accurate and might be caused by variations in nanoparticles properties (like charge, shape, size, coating) and use of different quantification methods [20]. Figure 2 explains the nanoparticle attributes influencing drug delivery via the intranasal route.

TABLE 1

Q6 Impact of various structural characteristics of nasal cavity on permeation

Structural parts/region of the nasal cavity

Nasal hairs (vibrissae) of the nasal vestibule (sebaceous glands)

Transepithelial region of the atrium (narrowest region)

Superior, middle and inferior turbinate of the respiratory region

Specialized ciliated olfactory nerve cells of the olfactory region Ciliated cells and squamous epithelial cells of the nasopharynx

\section{Effect on permeation}

Least permeable owing to the presence of keratinized cells

Less permeable because it has a small surface area and stratified cells are present anteriorly

Most permeable region due to greater surface area and increased blood supply

Direct pathway to cerebrospinal fluid (CSF) Nasal cavity drainage receiver 


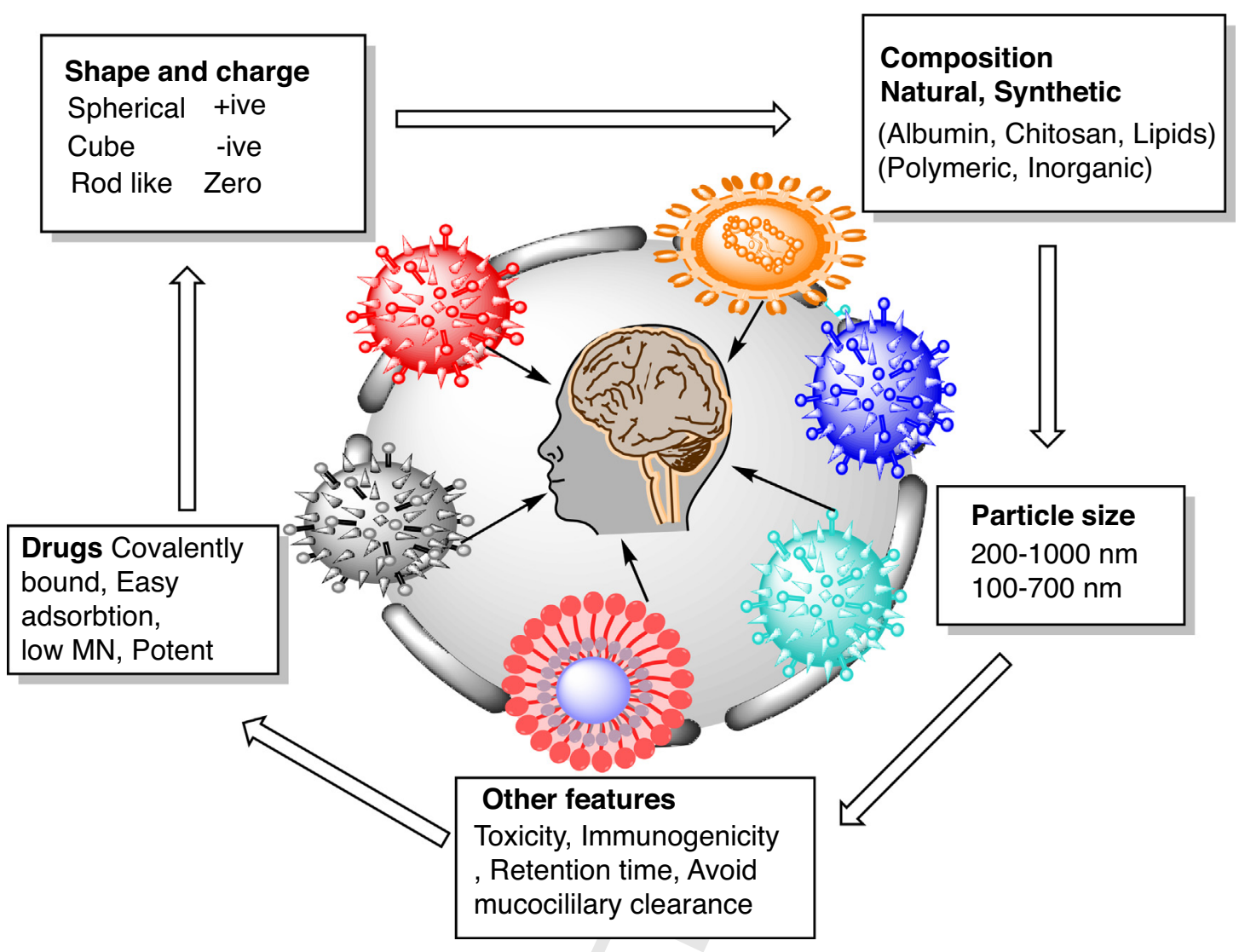

$\overline{\text { Drug Discovery Today }}$

FIGURE 2

Q2 Nanoparticle attributes influencing the delivery of API via the intranasal route. The figure illustrates the significant features of nanoparticles for intranasal delivery like pysiochemical properties (size, charge, composition) and other significant features such as low immunogenicity, low toxicity, avoiding mucociliary Q3 clearance and increased retention time (high mucoadhesivity).

\section{Lipid nanocarriers: a promising approach for intranasal delivery}

For significant nose-to-brain delivery, a colloidal drug delivery system is the most suitable system that refers to increased bioavailability and sustained release and high stability of the API. The enhanced biocompatibility, high scalability, safety and efficacy of lipid nanoparticles made them superior carriers for nose-to-brain Q22 delivery. In contrast to lipid nanoparticles, polymeric nanoparticles have low scalability, high cytotoxicity (e.g., 100\% mortality was reported when cells were treated with polyester polymer nanoparticles) and poor tolerability. Most lipid nanoparticles have a particle size within the range $50-1000 \mathrm{~nm}$. As described in the previous section, a particle size between 50 and $700 \mathrm{~nm}$ is the most favorable for intranasal delivery via neuronal transport [15]. Among these lipid carriers, liposomes and SLNs discussed in the following section have shown greater therapeutic efficacy in GBM. The lipid nanoformulations of anticancer agents provide enhanced drug stability, PK, drug distribution and efficacy compared with other approaches [7].

\section{Therapeutic efficacy of lipid nanoparticles in targeting glioblastoma}

Liposomes, SLNs, lipoproteins, lipoplexes and nanostructured lipid carriers have been widely used for targeting glioblastoma.
Liposomes are bilayer spherical biocompatible carriers and these lipid-based nanostructures are actually the pioneers of lipid-based particles employed for parenteral delivery with diameter ranges from 10 to $1000 \mathrm{~nm}$ and are precursors of NLCs and SLNs [21]. Q23 Table 2 summarizes the use of liposomes in the management of MGs via different delivery routes. The results of all these studies revealed an increase in survival time along with the inhibition of proliferation [22]. It should be considered that various liposomal factors like the size, particle diameter and uptake by the MPS can be crucial for targeting [23].

Lipid nanoparticles including SLNs and NLCs are the most suitable among all other lipid nanoparticles, without being constrained by their limitations. The stealth ability of lipid nanoparticles is higher than other polymeric nanoparticles against the MPS because they are fabricated from biocompatible substances such as a brew of natural lipids [24]. Table 3 demonstrates the therapeutic efficacy of SLNs and NLCs in targeting glioblastoma. The results of all previous studies revealed the decrease in tumor growth, the increase in the lifespan of the animals used and the inhibition of cell proliferation.

The proven efficacy (from studies mentioned in Tables 2 and 3) of lipid nanoparticles in targeting glioblastoma has shown their potential to encapsulate anticancer drugs. Furthermore, these lipid nanoparticles have a good ability to protect the loaded API and, 
TABLE 2

Applications of liposomes in the management of glioblastoma multiforme (GBM)

Encapsulated substance

Model of study

Type of liposomes

Clinical study

PEG-liposomes

Doxorubicin

Clinical study

Cationic liposomes

Plasmid

Recombinent herpes

simplex

virus thymidines kinase

(adeno viral carrier)

Antisense growth factor

Lomustine

Q7 EPI plus celecoxib

Q8 PTX

Glioma model in

Cationic liposomes

mouse

Human Cationic liposomes

malignant glioma

cell lines

Rabit glioma

model

Mice

Mice

Mice

SiRNA

Mice

DNR and quinacrine

Mice

Rats

Rats
Temperature-sensitive liposomes (TSL)

PTD peptide attached liposomes

Dual targeting, cell penetrating

peptid attached liposomes

Ligand-targeted liposomes (CTX)

Ligand-targeted liposomes

(WGA and TAM)

Ligand-targeted liposomes (RGD)

Ligand-targeted liposomes

Lactoferrin liposomes
Central nervous system (CNS) action/effects on GBM

Enhanced efficacy

Inhibition of tumor growth

Enhanced survival time

Antiproliferative

Reduction of tumor size

Reduced immunogenicity

Antiproliferative

Inhibition of tumor growth

Thermo targeting with inhibition of tumor growth

Destruction of glioma vasculogenic mimicry channels

Selective targeting

Inhibition of tumor growth

Enhance the efficacy and internalization into glioma

cells

Killing of glioblastoma cells and dimishing brain gliomas

Enhance targeting ability and site-specific delivery

Inhibit tumor growth increase lifespan of rats

Destruction of tumor cells and significant enhanced survival rate in tumor-bearing rats
Refs

TABLE 3

Summary of applications of SLNs/NLCs in targeting glioblastoma multiforme (GBM)

\begin{tabular}{|c|c|c|c|c|}
\hline Encapsulated substance & Model of study & Type of SLN/NLCs & $\begin{array}{l}\text { Central nervous system (CNS) action/ } \\
\text { effects on GBM }\end{array}$ & Refs \\
\hline armustine & U87 cell line (In vitro human brain model) & $\begin{array}{l}\text { Cationic solid lipid nanoparticles } \\
\text { (CASLNs) }\end{array}$ & $\begin{array}{l}\text { Antiproliferative effect } \\
\text { Decrease expression of tumor necrosis } \\
\text { factor (TNF)- } \alpha\end{array}$ & 48] \\
\hline Doxorubicin and etoposide & U87 cell lines, HBMEC, human astrocytes & CASLNs & Significant reduction in tumor growth & [49] \\
\hline Edelfosine (EDF) & $\begin{array}{l}\text { Glioma cell line C6 in vivo C6 glioma } \\
\text { xenograft tumor }\end{array}$ & SLNs (composed of compritolor) & $\begin{array}{l}\text { The antimalignant effect, inhibition of } \\
\text { tumor growth }\end{array}$ & {$[50]$} \\
\hline Cytarabine (CRB) & EL-4 cell lines & NLCs & Cytotoxic effect on tumor cell line & {$[51]$} \\
\hline siRNAs & $\begin{array}{l}\text { U87MG cell lines and tumor xenograft for in } \\
\text { vivo study }\end{array}$ & $\begin{array}{l}\text { Low-density lipoprtoein (LDL) } \\
\text { and polyethylene glycol (PEG) } \\
\text { SLNs }\end{array}$ & The decrease in tumor cell proliferation & {$[52]$} \\
\hline Camptothecin (CPT) & $\begin{array}{l}\text { BCEC Porcine brain capillary endothelial } \\
\text { cells compared with (RAW264.7) }\end{array}$ & CA-SLNs & $\begin{array}{l}\text { Higher cytotoxicity in brain cells } \\
\text { enhance antitumor efficacy }\end{array}$ & {$[53]$} \\
\hline Etoposide & $\begin{array}{l}\text { K562 cell line, MTT assay and flow } \\
\text { cytometry }\end{array}$ & NLCS with transferrin & $\begin{array}{l}\text { Enhanced cellular uptake and } \\
\text { antiproliferative effect }\end{array}$ & [54] \\
\hline $\begin{array}{l}\text { Locked nucleic acid (LNA) } \\
\text { (antioncogenic miR-21) }\end{array}$ & U87 MG (malignant glioma cell line) & $\begin{array}{l}\text { Lipid nanocapsule (LNCs) with } \\
\text { L-1 peptide }\end{array}$ & $\begin{array}{l}\text { Reduction of miR-21 expression and } \\
\text { antiproliferative }\end{array}$ & {$[55]$} \\
\hline Curcumin & U251MG cell line, rats bearing C6 gliomas & CA-LNCS & $\begin{array}{l}\text { The decrease in tumor size and } \\
\text { malignancy }\end{array}$ & {$[56]$} \\
\hline Resveratrol (RVR) & ine & Functiona & Enhance cytotoxicity & {$[57]$} \\
\hline Doxorubicin (DOX) & BBB model (hcmec/D3 cell) & CA-SLNs & Increased toxicity for glioblastoma cells & {$[58]$} \\
\hline $\begin{array}{l}\text { Polo-like kinase } 1 \text { (PLK1) } \\
\text { siRNAs (siPLK1) }\end{array}$ & Rats bearing orthotopic xenograft model & HA-LNPs (hyaluronic acid) & $\begin{array}{l}\text { Increased cell death (by reducing } \\
\text { expression of PLK1) }\end{array}$ & {$[59]$} \\
\hline Temozolomide (TMZ) & U87MG in vitro cells lines & NLCs & Very much enhanced antitumor activity & {$[60]$} \\
\hline Vincristine (VCR) and TMZ & $\begin{array}{l}\text { U87MG in vitro cell line and mice induced } \\
\text { with malignant glioma model }\end{array}$ & SLNs and NLCs & $\begin{array}{l}\text { NLCS show better antitumor activity } \\
\text { than SLNs }\end{array}$ & {$[61]$} \\
\hline
\end{tabular}

because of their occlusive nature, they can also increase nasal retention time. These features make lipid nanoparticles a significant carrier for nose-to-brain delivery because they can avoid the cytotoxicity issues of antineoplastic drugs $[7,25]$.

\section{Novel lipid nanoparticle formulation for targeting glioblastoma via IND}

New delivery approaches are required in research to efficiently target brain tumors. Curcumin-loaded NLCs (CUR-NLC) for 
intranasal administration were developed with a particle diameter of $146 \mathrm{~nm}$, encapsulation efficiency (EE) of 90\%, a charge of $21 \mathrm{mV}$ and polydispersity index (PDI) rating of 0.18 . The results of the following investigation reveal the increased cytotoxicity of CUR-NLC compared with that of free CUR in the glioma cell line U373MG. The biodistribution study for the same formulation showed an increased drug concentration in the brain after the intranasal application of NLCs. The results of this study led to the conclusion that CUR-NLC is an efficient delivery system for targeting glioblastoma [23]. Temozolomide-loaded NLCs (TMZ-NLC) were prepared to ensure brain targeting via the intranasal route. The optimized formulation showed a particle size within the nano range, zeta potential of $15 \mathrm{mV}$, entrapment efficiency of $81 \%$ and PDI of 0.2 . The results of the in vivo studies indicated the significant enhanced brain concentration of TMZ-NLC in comparison with TMZ dispersion (i.v., intranasal). The highest concentration of TMZ-NLC in the brain proved the efficacy of this direct intranasal administration of NLCs. The following study described that the intranasal administration of NLCs increased residence time and resulted in higher bioavailability in the brain at lower doses, denoting this delivery route the most suitable for targeting glioma [26].

Novel farnesyl thio salicylic acid (FTA)-loaded lipid cationic hybrid nanoparticles (HNPs) were formulated and evaluated for antitumor activity via the intranasal route. Glioma 2 (RG2) cells were placed into Wistar rats. The tumor-bearing rats were treated with FTA-encapsulated HNPs by intranasal and i.v. administration. The evaluation of tumor sizes with FTA-encapsulated HNPs resulted in a clear decrease $(\sim 55 \%)$ in tumor size. This study proved that the intranasal intervention of FTA-loaded HNPs is an equally effective approach in glioblastoma targeting. The results of all studies support the use of the IND route for targeting glioblastoma by using lipid carriers [27].

\section{Mechanism of nanoparticle drug delivery via the intranasal route}

The intranasally administered formulation will deposit on the pseudostratified columnar epithelium (a respiratory tract in the nasal cavity). The site of deposition of the intranasal formulation administered in the form of solution, spray or gel (via applicator) is the front region of the nasal cavity. However, there are a few devices that can settle the drug formulation in a higher region of the nasal cavity. The nanoparticles intervene via intranasal passage deposited at the site of the nasal cavity depending on its properties like size, charge and lipophilicity. There are four options for the drug, either to enter via the nasal epithelial tissue and arrive at the circulation or be unloaded along the gastrointestinal tract (GIT) through the nasopharynx by the ciliary clearance network. The system is made up of cilia, which are motile and beat in a synchronized manner, thereby propelling the viscous superior part dorsally against the nasopharynx quickly ( $5 \mathrm{~mm} / \mathrm{min}$ ). In addition, enzymatic activity is also higher in the nasal cavity deep in the olfactory region (enzymes like cytochrome P450 dependent peptidases, monooxygenase and proteases are involved in that procedure) [28]. The nose-to-brain delivery of the nanoparticles will follow the transport from endothelial cells to the olfactory neurons via endocytosis or pinocytosis and move along the axon or it will follow the trigeminal nerve pathway. For this transport pathway, the size of nanoparticles should be within the diameter of the axon: 100-700 nm [29]. Figure 3 explains all the possible mechanisms and pathways involved in the transport of lipid nanoparticles.

\section{In vitro/in vivo/ex vivo models for testing nose-to-brain delivery}

For an exploration of the mechanism behind the transport of drugs via the intranasal route, different types of in vitro, in vivo and ex vivo models are used. These diverse types of models are applied for different studies: in vitro models for permeation and diffusion studies; in vivo models for the determination of absorption and PK profile of the API; and ex vivo models for perfusion studies in the nasal cavity. The selection of in vivo models should be adequate for studying the anatomy of the nasal cavity. The first animal model used for intranasal study was the rat and, later, with the development in absorption data, other animal models like sheep, monkey, mouse and rabbit were also used. For adequate PK studies rabbit, dog, sheep and monkey models are commonly suggested, whereas mouse and rat models are used for preliminary absorption studies [30].

Besides the significance of in vivo models, the transport mechanism of drug absorption from the nasal route had to be explored. In vitro models were fabricated to replace the in vivo and ex vivo models. Furthermore, it is difficult to extrapolate the data of the absorption and kinetics studies obtained from the animal models to humans (owing to the difference of species). It is necessary to select adequate cell lines that can reproduce results at significantly low costs. There are a number of in vitro cell culture models like NAS2BL (originating from rat nasal squamous carcinoma), BT (originating from bovine turbinates), CaCo-2 cell lines (from human colon carcinoma), Calu-3 (originating from human lung adenocarcinoma), RPMI 2650 (from human nasal epithelial tissues) and 16HBE14o(from human normal bronchial epithelium of male heart lung transplant patient) [30]. Among these models, CaCo-2 and RPMI 2650 are used to evaluate permeability and absorption via the nasal route. However, there are some disadvantages of cell lines, for example RPMI 2650 are undifferentiated cells that encounter the limited expression of ciliated and goblet cells. The absence of a developed monolayer makes this cell line impractical to use for a transport study. By contrast, Calu3 cells can develop monolayers and are suitable for transport study but the origin of this cell line is not the normal epithelial cells of the nasal cavity. The 16HBE140-cell line possesses high transepithelial electrical resistance (TEER) making it suitable for transportation study but this cell line originates from normal bronchial epithelium of a male heart and lung transplant patient. For the determination of drug delivery and the development of formulations via the intranasal route, it is important to use reliable ex vivo models. The excised tissue is usually from the nasal mucosa of slaughtered or experimental animals (rats, rabbits, dogs, monkeys, sheep) or from humans as well. The ex vivo study is very important to obtain 


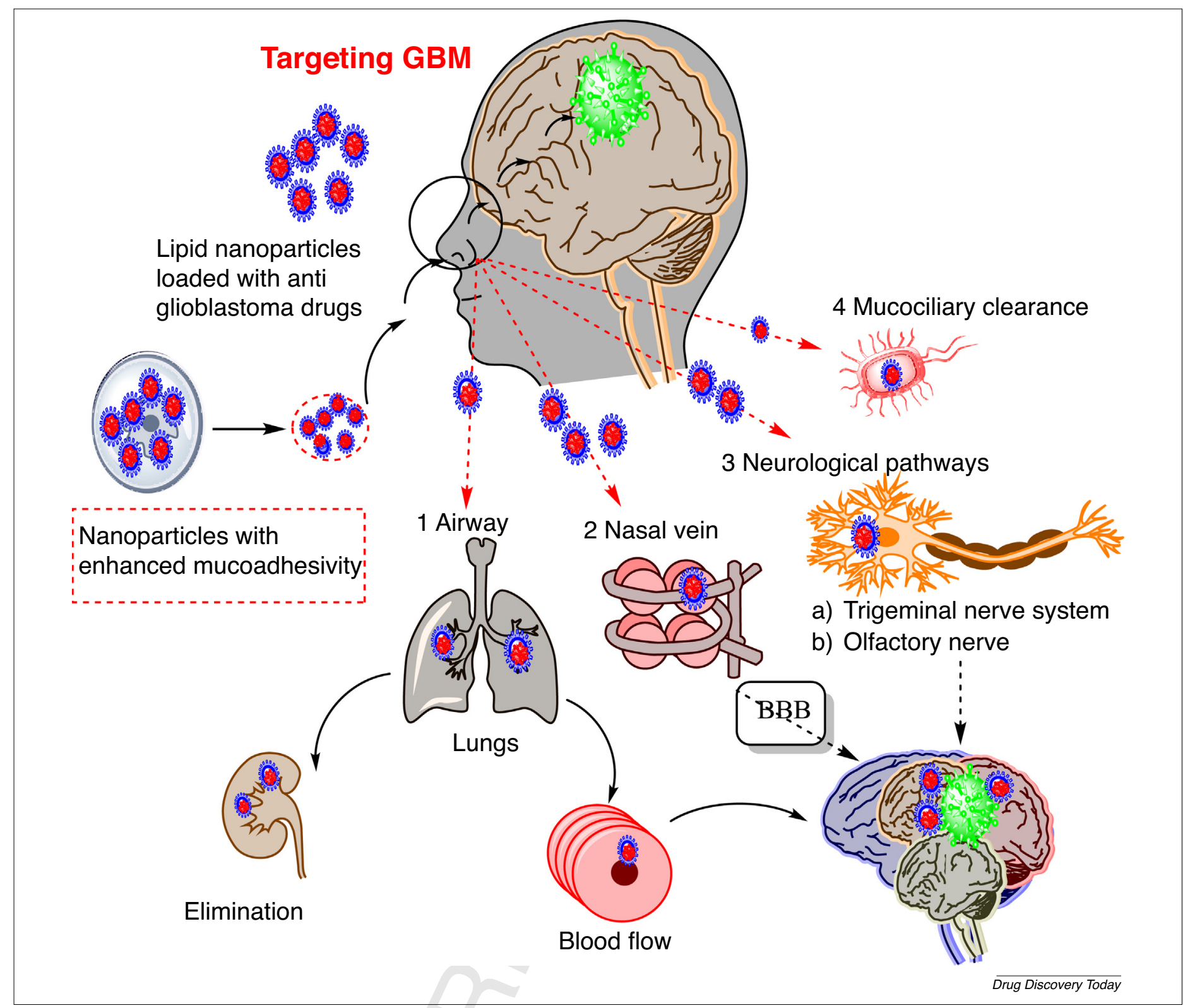

FIGURE 3

Q4 Possible mechanisms of lipid nanoparticles across the nasal membrane. The figure demonstrates that lipid nanoparticles with enhanced mucoadhesivity will follow four possible pathways: (i) airway; (ii) nasal vein; (iii) neurological pathway; and (iv) mucociliary clearance. The nanoparticles will follow the transport from endothelial cells to olfactory neurons via endocytosis or pinocytosis and move along the axon or follow the trigeminal nerve pathway.

all the information regarding the toxicity, efflux, metabolism and permeation of the developed formulation. Besides the many advantages of ex vivo models, there are a few limitations, such as the lack of interstitial flow rate determination and the thickness of nasal epithelial tissues of the excised mucosa. The Ussing chamber is the ex vivo nasal model for permeability studies [31].

\section{Significance of QbD in the early development of a lipid carrier system for targeting glioblastoma}

Depending on up-to-date knowledge about barriers limiting the IND of anticancer drugs targeting brain tumors, lipid-based nanocarrier systems could offer a promising strategy. However, because many formulation parameters and regulatory aspects should be considered, the QbD concept or the GMP of the 21st century should be followed. The main elements of QbD methodology are described in the relevant guidelines of the International Council on Harmonization (ICH), specifically ICH Q8 (R2), Q9 and Q10; and these include: (i) defining the quality target product profile (QTPP); (ii) selecting the critical quality attributes (CQAs) of the targeted product; (iii) selecting the production method and defining the critical process parameters (CPPs) that can highly affect the CQAs; and (iv) analysis of the initial risk assessment (RA), which is followed by optimizing the level of the risky factors by applying a suitable design of experiment (DOE) [32]. The very first step of $\mathrm{QbD}$ is to collect all the data from previous studies that could affect 


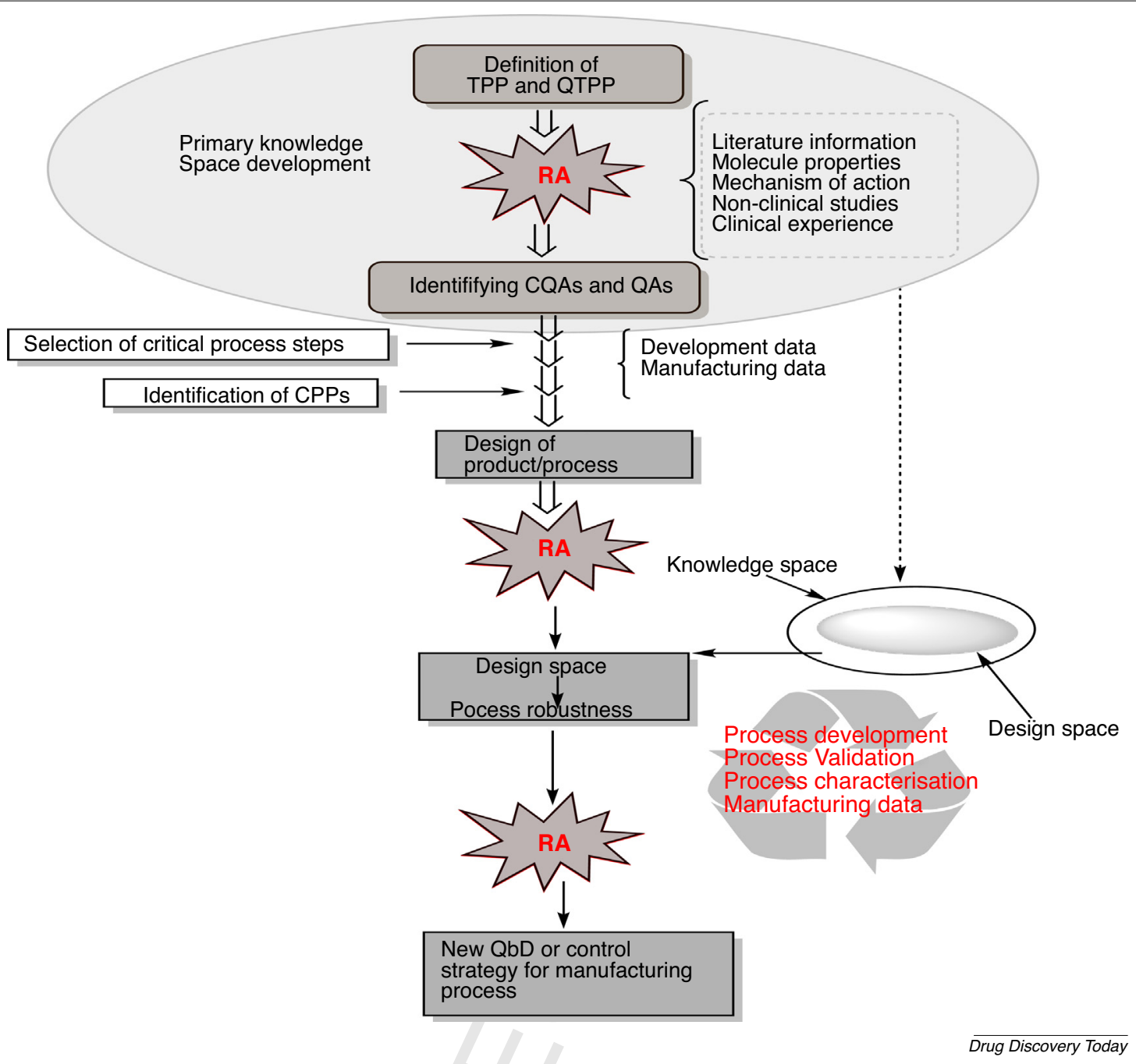

FIGURE 4

Q5 Steps and elements of quality by design (QbD) methodology that will help to design and validate the process for development of intranasal formulation. Step 1: identification of target product profile (TPP) and quality target product profile (QTPP), which comprises therapeutic and other quality requirements. Step 2: identification of critical quality attributes (CQAs), which are associated with in-process materials, and critical process parameters (CPPs) having an effect on CQAs. Step 3: risk assessment (RA) is a process of collecting information to support risk decision and it is also a main activity of QbD methodology that can be performed at initial and final phases of development.

the target product profile. After early knowledge of space design and evaluation of QTPPS, CQAs and CPPs, the RA application revealed the attributes having the highest impact on the final lipid nanoformulation quality for IND. Figure 4 demonstrated the description of QbD methodology in early development of a lipid carrier system for nose-to-brain delivery based on the relevant ICH guidelines $[33,34]$.

\section{Concluding remarks and future directions}

This review summarizes the importance of nose-to-brain delivery in targeting GBM. Nanoformulations are considered as one of the most important targeting carriers. Besides many advantages, nanoformulations loaded with cytotoxic material can still accumulate in other parts and tissues of the body, for instance in the liver, spleen and kidney. Therefore, it is necessary to design and fabricate a method that can overcome the shortcomings of previously used carriers and delivery routes. The alternative route and the lipid nanocarrier provide chances to deliver anticancer drugs (with potential efficacy) against GBM, and this will be a new and expedient approach to GBM treatment strategies. Furthermore, for successful IND of anticancer drugs, risk assessment is the main component of QbD which should be applied using special software to calculate the risk severity of CQAs and CPPs regarding the encapsulation of potential API into lipid-based carrier systems. Thus, the application of the QbD concept can save time and effort by directing the focus toward structuring the quality in each step of formulation design.

\section{Conflicts of interest}

The authors declare no conflicts of interest, financial or otherwise. Q24 


\section{Acknowledgments}

F.S. made substantial contributions to writing the manuscript and designing the figures and tables. R.I. contributed to evaluating the manuscript, writing the $\mathrm{QbD}$ and general opinion sections, in addition to making critical revision of the whole manuscript. I.C. contributed to the conception of the manuscript, planning and supervising the work at each step and giving the final approval.

\section{References}

1 Hombach-Klonisch, S. et al. (2018) Glioblastoma and chemoresistance to alkylating agents: involvement of apoptosis, autophagy, and unfolded protein response. Pharmacol. Ther. 184, 13-41

2 van Schaijik, B. et al. (2019) Circulating tumor stem cells and glioblastoma: a review. J. Clin. Neurosci. 61, 5-9

3 Zhang, Q. et al. (2018) Current status and potential challenges of mesenchymal stem cell-based therapy for malignant gliomas. Stem Cell Res. Ther. 9, 1-9

4 Mughal, A.A. et al. (2018) Patterns of invasive growth in malignant gliomas the hippocampus emerges as an invasion-spared brain region. Neoplasia 20, 643-656

5 Sekerdag, E. et al. (2017) Nose-to-brain delivery of farnesylthiosalicylic acid loaded hybrid nanoparticles in the treatment of glioblastoma. J. Neurol. Sci. 381, 171-172

6 Tucker, C. et al. (2018) The intranasal route as an alternative method of medication administration. Crit. Care Nurse 38, 26-31

7 Battaglia, L. et al. (2018) Lipid nanoparticles for intranasal administration: application to nose-to-brain delivery. Expert Opin. Drug Deliv. 15, 369-378

8 Shankar, R. et al. (2018) Lipid nanoparticles: a novel approach for brain targeting. Pharm. Nanotechnol. 6, 81-93

9 Lakkadwala, S. and Singh, J. (2019) Co-delivery of doxorubicin and erlotinib through liposomal nanoparticles for glioblastoma tumor regression using an in vitro brain tumor model. Colloids Surf. B Biointerfaces 173, 27-35

10 Shah, M.K. et al. (2019) Lipid nanocarriers: preparation, characterization and absorption mechanism and applications to improve oral bioavailability of poorly water-soluble drugs. Biomed. Appl. Nanoparticles 2019, 117-147

11 Glaser, T. et al. (2017) Targeted nanotechnology in glioblastoma multiforme. Front. Pharmacol. 8, 166

$12 \mathrm{He}$ Q. et al. (2018) Towards improvements for penetrating the blood-brain barrier - recent progress from a material and pharmaceutical perspective. Cells 7, 24

13 Pires, P.C. and Santos, A.O. (2018) Nanosystems in nose-to-brain drug delivery: a review of non-clinical brain targeting studies. J. Control. Release 270, 89-100

$14 \mathrm{Md}$, S. et al. (2018) Nano-carrier enabled drug delivery systems for nose to brain targeting for the treatment of neurodegenerative disorders. J. Drug Deliv. Sci. Technol. 43, 295-310

15 Selvaraj, K. et al. (2018) Nose to brain transport pathways an overview: potential of nanostructured lipid carriers in nose to brain targeting. Artif. Cells Nanomed. Biotechnol. 46, 2088-2095

16 Mistry, A. et al. (2009) Effect of physicochemical properties on intranasal nanoparticle transit into murine olfactory epithelium. J. Drug Target 17, $543-552$

Q25 17 Schwarz, B. and Merkel, O.M. (2019) Nose-to-brain delivery of biologics. Future Sci. http://dx.doi.org/10.4155/tde-2019-0013

18 Pallagi, E. et al. (2018) Initial risk assessment as part of the quality by design in peptide drug containing formulation development. Eur. J. Pharm. Sci. 122, 160-169

19 Qindeel, M. et al. (2019) Development of novel pH-sensitive nanoparticles loaded hydrogel for transdermal drug delivery. Drug Dev. Ind. Pharm. 45, 1-29

20 Rizvi, S.A. and Saleh, A.M. (2018) Applications of nanoparticle systems in drug delivery technology. Saudi Pharm. J. 26, 64-70

21 Liao, W.etal. (2018) Recent advances on glioblastoma multiforme and nano-drug carriers: a review. Curr. Med. Chem. . http://dx.doi.org/10.2174/0929867325666180514113136

22 Riaz, M. et al. (2018) Surface functionalization and targeting strategies of liposomes in solid tumor therapy: a review. Int. J. Mol. Sci. 19, 195

23 Madane, R.G. and Mahajan, H.S. (2016) Curcumin-loaded nanostructured lipid carriers (NLCs) for nasal administration: design, characterization, and in vivo study. Drug Deliv. 23, 1326-1334

24 Anand, A. et al. (2019) Brain targeted delivery of anticancer drugs: prospective approach using solid lipid nanoparticles. IET Nanobiotechnol. 13 353-362

25 Sandoval, M.A. et al. (2012) EGFR-targeted stearoyl gemcitabine nanoparticles show enhanced anti-tumor activity. I. Control. Release 157, 287-296

26 Khan, A. et al. (2016) Brain targeting of temozolomide via the intranasal route using lipid-based nanoparticles: brain pharmacokinetic and scintigraphic analyses. Mol. Pharm. 13, 3773-3782

27 Sekerdag, E. et al. (2017) A potential non-invasive glioblastoma treatment: nose-to-brain delivery of farnesylthiosalicylic acid incorporated hybrid nanoparticles. J. Control. Release 261, 187-198

28 Crowe, T.P. et al. (2018) Mechanism of intranasal drug delivery directly to the brain. Life Sci. 195, 44-52

29 Samaridou, E. and Alonso, M.J. (2018) Nose-to-brain peptide delivery - the potential of nanotechnology. Bioorg. Med. Chem. 26, 2888-2905

30 Sousa, F. and Castro, P. (2016) Cell-based in vitro models for nasal permeability studies. In Concepts and Models for Drug Permeability Studies (Sarmento, B., ed.), pp. 83-100, Elsevier

31 Erdö, F. et al. (2018) Evaluation of intranasal delivery route of drug administration for brain targeting. Brain Res. Bull. 143, 155-170

32 Nayak, A.K. et al. (2019) Application of quality by design for the development of biopharmaceuticals. In Pharmaceutical Quality by Design. pp. 399-411, Elsevier

33 Csóka, I. et al. (2018) Extension of quality-by-design concept to the early development phase of pharmaceutical R\&D processes. Drug Discov. Today 23, 1340-1343

34 Pallagi, E. et al. (2018) Initial risk assessment as part of the quality by design in peptide drug containing formulation development. Eur. J. Pharm. Sci. 122, 160-169

35 Saudagar, R. and Kulkarni, M.M. (2017) Review on in-situ nasal gel drug delivery system. Res. J. Pharm. Technol. 10, 1870

36 Palhal, A.P. et al. (2017) In-situ nasal gel: modernistic advancement in drug delivery.. Q28 2017. http://dx.doi.org/10.20959/wjpr201711-9512

37 Beier, C.P. et al. (2009) RNOP-09: pegylated liposomal doxorubicine and prolonged temozolomide in addition to radiotherapy in newly diagnosed glioblastoma - a Phase II study. BMC Cancer 9, 308

38 Yoshida, J. et al. (2004) Human gene therapy for malignant gliomas (glioblastoma multiforme and anaplastic astrocytoma) by in vivo transduction with human interferon $\beta$ gene using cationic liposomes. Human Gene Ther. 15, 77-86

39 Jahangiri, A. et al. (2017) Convection-enhanced delivery in glioblastoma: a review of preclinical and clinical studies. J. Neurosurg. 126, 191-200

40 Aigner, A. and Kögel, D. (2018) Nanoparticle/siRNA-based therapy strategies in glioma: which nanoparticles, which siRNAs? Nanomedicine 13, 89-103

41 Song, J. et al. (2018) Effectiveness of lomustine and bevacizumab in progressive glioblastoma: a meta-analysis. OncoTarget. Ther. 11, 3435

42 Ju, R.-J. et al. (2016) Destruction of vasculogenic mimicry channels by targeting epirubicin plus celecoxib liposomes in treatment of brain glioma. Int. J. Nanomed. 11,1131

43 Liu, Y. et al. (2016) Dual receptor recognizing cell penetrating peptide modified liposomes for anti-glioma therapy. Nanomed. Nanotechnol. Biol. Med. 2, 500

$44 \mathrm{Li}, \mathrm{X} .-\mathrm{T}$. et al. (2014) Multifunctional targeting daunorubicin plus quinacrine liposomes, modified by wheat germ agglutinin and tamoxifen, for treating brain glioma and glioma stem cells. Oncotarget 5, 6497

45 Pacheco-Torres, J. et al. (2015) Image guided drug release from $\mathrm{pH}$-sensitive ion channel-functionalized stealth liposomes into an in vivo glioblastoma model. Nanomed. Nanotechnol. Biol. Med. 11, 1345-1354

46 Chen, P.-Y. et al. (2012) Comparing routes of delivery for nanoliposomal irinotecan shows superior anti-tumor activity of local administration in treating intracranial glioblastoma xenografts. Neuro Oncol. 15, 189-197

47 Chen, H. et al. (2011) Lactoferrin modified doxorubicin-loaded procationic liposomes for the treatment of gliomas. Eur. J. Pharm. Sci. 44, 164-173

48 Kuo, Y.-C. and Liang, C.-T. (2011) Inhibition of human brain malignant glioblastoma cells using carmustine-loaded catanionic solid lipid nanoparticles with surface anti-epithelial growth factor receptor. Biomaterials $32,3340-3350$

49 Kuo, Y.-C. and Lee, C.-H. (2015) Inhibition against growth of glioblastoma multiforme in vitro using etoposide-loaded solid lipid nanoparticles with $\rho$-aminophenyl- $\alpha$-D-manno-pyranoside and folic acid. J. Pharm. Sci. 104 1804-1814 
50 Kuo, Y.-C. and Wang, C.-C. (2015) Carmustine-loaded catanionic solid lipid nanoparticles with serotonergic $1 \mathrm{~B}$ receptor subtype antagonist for in vitro targeted delivery to inhibit brain cancer growth. J. Taiwan Inst. Chem. Eng. 46, 1-14

51 Sharma, P. et al. (2011) Development and evaluation of nanostructured lipid carriers of cytarabine for treatment of meningeal leukemia. J. Nanosci. Nanotechnol. 11, $6676-6682$

52 Jin, J. et al. (2011) In vivo specific delivery of c-Met siRNA to glioblastoma using cationic solid lipid nanoparticles. Bioconj. Chem. 22, 2568-2572

53 Martins, S.M. et al. (2013) Brain targeting effect of camptothecin-loaded solid lipid nanoparticles in rat after intravenous administration. Eur. J. Pharm. Biopharm. 85, 488-502

54 Khajavinia, A. et al. (2012) Targeting etoposide to acute myelogenous leukaemia cells using nanostructured lipid carriers coated with transferrin. Nanotechnology 23 , 405101

55 Griveau, A. et al. (2013) Silencing of miR-21 by locked nucleic acid-lipid nanocapsule complexes sensitize human glioblastoma cells to radiation-induced cell death. Int. J. Pharm. 454, 765-774
56 Chen, Y. et al. (2016) Nanostructured lipid carriers enhance the bioavailability and brain cancer inhibitory efficacy of curcumin both in vitro and in vivo. Drug Deliv. 23, 1383-1392

57 Jose, S. et al. (2014) In vivo pharmacokinetics and biodistribution of resveratrolloaded solid lipid nanoparticles for brain delivery. Int. J. Pharm. 474, 6-13

58 Chirio, D. et al. (2014) Positive-charged solid lipid nanoparticles as paclitaxel drug delivery system in glioblastoma treatment. Eur. J. Pharm. Biopharm. 88 746-758

59 Cohen, Z.R. et al. (2015) Localized RNAi therapeutics of chemoresistant grade IV glioma using hyaluronan-grafted lipid-based nanoparticles. ACS Nano 9, $1581-1591$

60 Song, S. et al. (2016) Novel RGD containing, temozolomide-loading nanostructured lipid carriers for glioblastoma multiforme chemotherapy. Drug Deliv. 23, 1404-1408

$61 \mathrm{Wu}, \mathrm{M}$. et al. (2016) Vincristine and temozolomide combined chemotherapy for the treatment of glioma: a comparison of solid lipid nanoparticles and nanostructured lipid carriers for dual drugs delivery. Drug Deliv. 23, 2720-2725 